\title{
An optimized ensemble sensitivity climatology of Mediterranean intense cyclones
}

\author{
L. Garcies and V. Homar \\ Universitat de les Illes Balears, Dept. Física, Ctra. Valldemossa, km. 7.5, 07122, Palma de Mallorca, Spain
}

Received: 2 June 2010 - Revised: 22 October 2010 - Accepted: 4 November 2010 - Published: 2 December 2010

\begin{abstract}
A new approach was recently proposed to compute climatological statistical sensitivities. It was applied on an already available classification of Mediterranean intense cyclones. However, those sensitivity results are questionable due to the limited homogeneity of some cyclone classes, which severely hampers the application of the statistical sensitivity analysis technique. In this study, a new classification of Mediterranean intense cyclones is built with the aim of improving the reliability of the final climatological sensitivity results. In addition to implementing a regional classification and a subsequent division according to the preceding conditions that lead to cyclone formation, a cluster pruning is applied to maximize the homogeneity of the classes. The increased cluster homogeneity and the higher significance of the obtained sensitivity estimates are quantified. Furthermore, the improved representativeness of the sensitivity fields derived after cluster pruning is tested and compared to previous results by means of numerical experiments. Eventually, summary sensitivity fields highlight the European Atlantic coasts, central and western Europe, the central and western Mediterranean basin and north african lands as sensitive regions for the evolution of these Mediterranean high-impact systems. Although some outstanding improvements are confirmed in this study, a further verification experiments are needed to objectively verify the sensitivity results and build solid confidence on the method.
\end{abstract}

\section{Introduction}

Under the ever-growing need from the public and authorities in accountable and relevant weather predictions, multiple approaches are currently explored with the primary goal

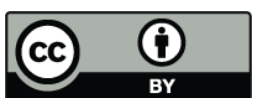

Correspondence to: L. Garcies

(lorena.garcies@uib.es) of improving forecast quality. One important research line is devoted to optimize the observing strategies to achieve better representations of the atmosphere and eventually more accurate forecasts. Numerical weather predictions are nowadays the primary raw material used in forecasting offices to issue short, mid and long term operational predictions, and all plans regarding the redesign of observational strategies should prioritize the optimization of numerical forecast capabilities. Sensitivity Analysis is a well matured technique that identifies causal atmospheric structures that have a relevant effect on a particular specified forecast aspect of interest. Therefore, information derived from such analysis could be very useful to provide guidance to executive actions regarding the design of an optimized observational network and special targeted observation strategies that would significantly improve the quality and value of numerical weather prediction (NWP) products.

EUCOS, which stands for EUMETNET Composite Observing System (http://www.eucos.net), is an operational EUMETNET programme whose main objective is the design and management of operational observations on a Europeanwide scale to improve NWP without increasing the overall cost. To accomplish this goal efficiently, information regarding the spatial and temporal distribution of forecast sensitivities should be available in a so called Climatology of Forecast Sensitivities. In this sense, Marseille and Bouttier (2000) showed that, over Europe and for all weather regimes, sensitivities of forecast errors are located mainly upstream of the westerlies, over the Northeastern Atlantic. However, the benefits of a redesign of a permanent observational network may be optimized by accounting for the socioeconomic impact of the events whose forecasts are to be improved. That is, remodeling decisions should take into special account hazardous weather episodes because of the larger potential benefits of the associated forecast improvements. To this end and according to the interest of the Mediterranean experiment on cyclones that produce high impact weather in

Published by Copernicus Publications on behalf of the European Geosciences Union. 
the Mediterranean (MEDEX, http://medex.inm.uib.es) in the design of an observational network that increases the accuracy of high impact weather forecasts, Homar et al. (2006), Homar et al. (2007) and Jansà and Homar (2006) contributed to the generation of an adjoint sensitivity climatology of Mediterranean Intense Cyclones using these systems as a proxy for high impact weather events in the Mediterranean basin. In fact, not all high impact events in the Mediterranean region are related to cyclones and most of the cyclones do not produce extreme weather; however, most Mediterranean high impact weather events are linked to cyclones (Jansà et al., 2001). Jansà and Homar (2006) showed by means of adjoint model runs that North Africa lands, the Mediterranean Sea and the Eastern North-Atlantic, which are poorly covered by in-situ observing networks, are relevant areas for the short-range forecast quality of Mediterranean intense cyclones. These sensitivity regions were also highlighted by the ensemble sensitivity climatology built by Garcies and Homar (2009) (hereafter, GH09). They showed that the evolution of these high-impact systems $24 \mathrm{~h}$ before the time of maturity depends largely on structures located over Western Europe, the Northern African lands and parts of east North Atlantic.

GH09 proposed a new climatological sensitivity technique based on the ensemble sensitivity method originally proposed by Hakim and Torn (2008). Ancell and Hakim (2007) defined ensemble sensitivity as the linear regression of analysis errors onto a given forecast metric, commonly referred to as response function $(J)$. A key fundamental point of the method proposed by GH09 is the lack of dependence on any ad hoc forecasting system as they applied the ensemble sensitivity approach not to ensembles of simulations but to the climatology of Mediterranean intense cyclones available from Campins et al. (2010). For each cyclone type, the raw sensitivity fields were obtained by linearly correlating the precursor conditions and the response function, which were defined as a measure of the cyclone's depth. The significance of the linear trends was accounted for by means of the correlation coefficient, filtering out irrelevant spurious trend values. Further, the final sensitivity fields were derived applying a normalization based on the local climatological variability of the precursor conditions. After all, the sensitivity results indicate expected variations on the depth of the cyclones produced by climatological standard perturbations applied to the precursor fields. As opposed to adjoint model based approximations, which rely on collections of individual case studies, this method renders sensitivity products which are statistically representative of a particular cyclone type. In addition, the computational cost of this technique is far less than that of the adjoint model runs.

The results obtained by GH09 for each Mediterranean intense cyclone type are easily interpretable both in amplitude and spatial distribution. Also, they are consistent with those obtained by Jansà and Homar (2006) using the adjoint model. However, the results of GH09 are questionable due to the limited homogeneity of some cyclone classes. In order to obtain representative sensitivity fields for each cyclone type, each initial ensemble of cyclones should univocally represent one cyclone class. Since GH09 made direct use of an already available classification of intense cyclones, their results can allegedly be improved by carefully refining the classification. In this study, we design an improved classification method oriented towards the application of ensemble sensitivities. The homogeneity and size of each cyclone class is optimized to improve the quality and reliability of the derived sensitivities.

This article is structured as follows: Section 2 documents the methodological procedure followed to achieve a classification of Mediterranean intense cyclones oriented to ensemble sensitivity. Section 3 is devoted to present sensitivity results obtained from the new classification and their derived benefits. Section 4 provides a summarized discussion of the methodological improvements and the final sensitivity fields.

\section{Clustering methodology}

The Mediterranean intense cyclone's database used in this study was built by Campins et al. (2010). This intense cyclone catalogue is based on the reanalysis fields from the European Center for Medium-Range Weather Forecast (ERA40, Uppala et al., 2005) and collects 1359 intense cyclones as those sea level depressions in the Mediterranean region detected over the 45-yr period covered by the ERA-40 (September 1957 to August 2002) with a maximum circulation exceeding $710^{7} \mathrm{~m}^{2} \mathrm{~s}^{-1}$ and a lifetime of at least $24 \mathrm{~h}$ (Campins et al., 2006). This study aims at improving the classification of these cyclones for better implementation of the Hakim and Torn (2008) statistical sensitivity analysis technique. More homogeneous clusters will better fulfill the hypothesis of small perturbations imposed by Ancell and Hakim (2007) for the results to be accurate and comparable to adjoint sensitivity fields. Eventually, the clustering process is designed to classify events by location of the mature cyclone and also by the preceding synoptic patterns. The following subsections expound on the details of the process leading to more homogeneous intense cyclone classes.

\subsection{Regional classification}

The aim of the first step of the clustering process is to join together those cyclones that achieved mature stage over the same region. According to the spatial distribution of cyclone's centers at the time of maximum intensity (Fig. 1), we select 11 regions with high density of intense mature cyclones: Western Mediterranean (Gulf of Lions and Balearic Sea), Algeria, Thyrrenian Sea, Adriatic Sea, Sicily, Ionian Sea, Aegean Sea, Sea of Crete, Cyprus, Turkey and Black Sea. Note that the maxima located over north Africa -mostly made up of large thermal summer lows- has been discarded for not being associated to hazardous weather from high vorticity or strong winds but fulfilling the constituting database 
circulation criteria due simply to size. The sample size of intense cyclones outside these areas is too small to derive significant statistical sensitivity products and are not further considered. In order to obtain a finer regional classification, we divide the two wider regions, that is, Western Mediterranean and Thyrrenian Sea into subclasses. To this end, we carry out a principal component analysis (PCA) over the mean sea level pressure field (MSLP) to ensure that only the most important modes of spatial variation in the field are considered by the clustering analysis (CA).

There are multiple possible modes for PCA (Richman, 1986), depending on the particular selection of variables, cases and fixed entity. We perform an S-mode PCA, that is, in the spatial domain. Indeed, S-mode PCA treats grid points as variables and days as observations. Note that this method is analogue to that applied in Jansà and Homar (2006) where a T-mode PCA and loadings as clustering variables were used instead. For the sake of spatial precision, only data from nearby regions is used to divide the two wide classes because PCA on larger windows encompassing much larger geographical areas can be unintendedly influenced by circulation features in remote regions. Another issue arises as how many principal components should be retained for the CA. Bearing in mind that the choice of the truncation level can be aided by many available principal component selection rules (Jollife, 2002) and it is ultimately a subjective choice depending on the data and the purpose of the CA, the number of principal components kept in the CA explains the $95 \%$ of the sample variance. Thus, it is expected to filter out the subscale noise and leave the relevant scales unaltered.

The CA used in this study is the non-hierarchical k-means classification algorithm (Anderberg, 1973). Among the nonhierarchical clustering approaches, which allow reassignment of observations as the analysis proceeds, k-means is the most widely used. The k-means method is named for the number of clusters into which the data will be grouped. Here, the purpose is to divide each of the two largest regions into two new subclasses. To this end, k-means CA is applied on the component scores obtained from PCA. In order to deal with the stochastic nature of the k-means results, a bootstrap strategy (1000 repetitions) is used. Then, a quantitative evaluation of the results of the bootstrap process is performed. Among the large number of cluster validation methods (Halkidi et al., 2001), we assess the quality of each partitioning computing the Dunn's index (Dunn, 1974) as it aims at the identification of compact and well separated clusters. Finally, the division with the largest value of the index is selected as the partitioning that best splits the cyclones on the Western Mediterranean and Thyrrenian Sea regions.

Eventually, the Western Mediterranean cluster is split into Gulf of Lions and Balearic Sea clusters. Similarly, the Tyrrhenian Sea cluster is divided into north and south cyclones. Thus, as a result of the regional classification, 753 Mediterranean intense cyclones are classified into 13 clusters (Fig. 1).

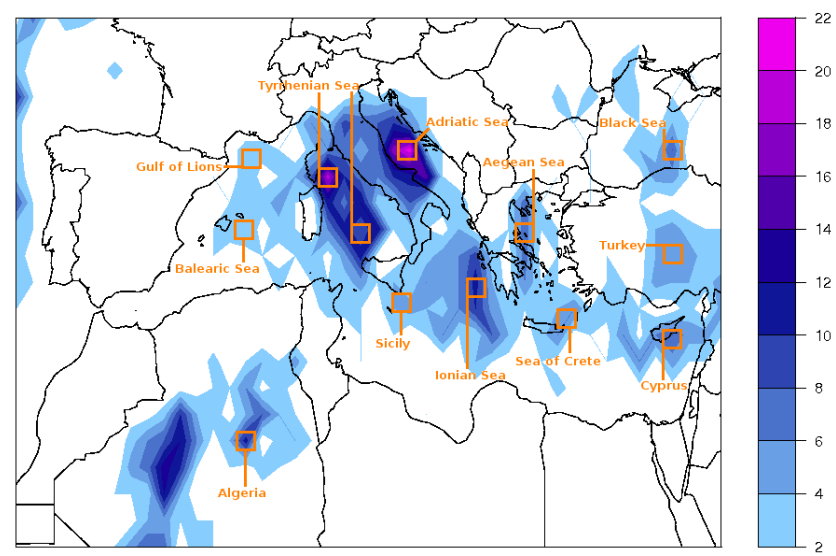

Fig. 1. Number of mature intense cyclones per square of $1.125^{\circ} \times$ $1.125^{\circ}$ over the $45-y r$ ERA-40 period. The labels indicate the location of the 13 selected regional cyclone classes.

\subsection{Subclassification considering precursor conditions}

After the first clustering step, those cyclones which achieve maturity over the same Mediterranean region are clustered together. However, this regional classification does not guarantee homogeneity over the preceding conditions that lead to their formation and so to similar sensitivity patterns. Therefore, a second round of classification taking into account precursor condition fields is needed to ensure representative sensitivity fields. Each of the 13 regional clusters is treated individually and subclassified considering precursor conditions back to $48 \mathrm{~h}$ before the time of maximum intensification of each cyclone (hereafter, $t$ ). The considered precursor conditions for the subclassification of the regional clusters are: geopotential height at $500 \mathrm{hPa}$ for $t-48 \mathrm{~h}$ and $t-24 \mathrm{~h}$, geopotential height at $200 \mathrm{hPa}$ for $t-48 \mathrm{~h}$ and temperature at $850 \mathrm{hPa}$ for $t-24 \mathrm{~h}$. It is noteworthy that mid-low levels of precursing fields have been used at $t-24 \mathrm{~h}$ due to their influence on the exact position of the mature surface cyclone during its last stages while mid-upper fields are relatively more influential at earlier stages $(t-48 \mathrm{~h})$. Again, PCA is also used to account for the main variation features of the selected fields and the principal components kept in the k-means calculations explains $95 \%$ of the field variance. Although there are multiple methods for estimating the appropiate number of clusters (Milligan and Cooper, 1985), none of the objective indexes that were tested in this second round of classification was useful to find a clear suggestion for each regional cluster, and so, a subjective method is adopted. Each regional cluster is divided from 1 (no division) to 4 subgroups following the same procedure explained in Sect. 2.1, that is, performing a bootstrap process evaluated by the Dunn's index. Then, all results from the multiple division of each regional cluster are analyzed by visual inspection and the most convenient subclassification is chosen. The decisions are made based on differences among the subclasses and the number of members 
in each one. This clustering step is very useful to remove outliers that are very easy to identify in the high order division tests. Those classes with very few members are discarded at this point of the process. As an example, the Balearic Sea regional cluster is made up of 41 members. The maximum Dunn's k-means CA that divides this cluster in 2 shows one subgroup with 39 members and the other with only 2 cyclones which have very different precursor conditions than those belonging to the more populated subgroup. Then, these 2 outliers are discarded from the Balearic Sea cluster. Finally, 666 days are classified into 23 preceding synoptic configurations that produce Mediterranean intense cyclones. Although this might seem a somewhat large number of clusters which provides too much detail, it is convenient to keep in mind that the ultimate goal is to compute representative sensitivities for each cyclone type. At the expense of a reduced sample size, the larger the number of clusters the more homogeneous they can be and so the more reliable the derived sensitivity fields will be.

\subsection{Pruning process}

GH09 obtained an increasing fading-out of the resulting sensitivity fields as these were computed earlier from the mature cyclone because the longer times have larger corrections due to weaker linear correlations. As expected, non-linear effects become dominant as we move away from the forecast aspect of interest for which we compute a linear estimate of its sensitivity. This reveals the migration from the initial quasi-linear regime towards the eventually non-linear regime. Taking this into account, a further step in the clustering process is added in this study. Bearing in mind that the initial evolution of small-amplitude perturbations in atmospheric models is well approximated by a linear regime, we attempt to reduce the spread of precursor conditions of each cluster at $t-48 \mathrm{~h}$ by means of a cluster pruning. The pruning process is aimed at maximizing the cluster density (i.e., number of cases per unit volume in the principal components space). The cluster density is proportional to the number of cluster members and inversely proportional to the cube of the euclidean distance between $500 \mathrm{hPa}$ geopotencial height fields of the cluster centroid and the farthest member. In general, for all clusters, the relationship between cluster density and the number of cluster members follows a common pattern (Fig. 2). As the number of events increases, so does the cluster density until it reaches a maximum. Then, the cluster density decreases because the added days are too distant and detrimental to the cluster density. Therefore, after cluster pruning, e.g. taking the number of members that maximizes density, the remaining cluster is the most homogeneous group of episodes representing a particular cyclone class in our database.

All clusters obtained at the subclassification stage (Sect. 2.2) are pruned. Consequently, 260 days are removed from these clusters and 406 episodes of Mediterranean in-

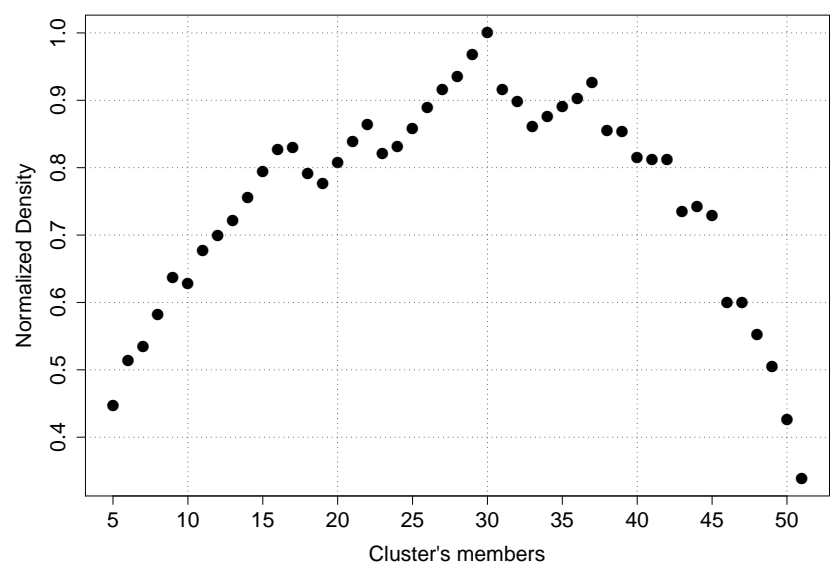

Fig. 2. Common pattern of normalized cluster density as a function of the number of events that make up a cluster. Example for an Adriatic Sea cluster: the pruned cluster is made up of 30 members which maximize the cluster density.

tense cyclones remain classified into 23 clusters (Table 1). This averages nearly 18 cyclones per class which is a moderate number of cases from a climatological perspective but a competitive amount when considering the original sensitivity calculation technique (Hakim and Torn, 2008) and compare it to typical weather forecast ensemble sizes. Admittedly, groups with less than 10 cases are very likely to undersample the subspace of relevant precursing perturbations leading to that cyclone class and so to missrepresent the corresponding sensitivity fields. However, the aforementioned careful distillation of homogeneous groups selects the optimum representatives of each cyclone type and so the derived sensitivity fields ought to -at least- sketch the main characteristics of the real sensitivity field.

\section{Sensitivity Results}

For each of the 23 clusters listed in Table 1, sensitivity fields are calculated following the methodology proposed by GH09. In particular, the precursor conditions are linearly correlated with the response functions and then a correction factor -which depends on the correlation coefficient- and a final normalization -based on the standard deviation of the precursor conditions- is applied. It is noteworthy that in this study we not only enhance the classification of Mediterranean intense cyclones used in the sensitivity analyses but we also add some improvements to the sensitivity computations. The precursor conditions considered are: temperature $(200,500$ and $850 \mathrm{hPa})$, wind speed $(200,500$ and $850 \mathrm{~Pa})$, geopotential height $(200,500,850$ and $1000 \mathrm{~Pa})$ and also the relative humidity $(850 \mathrm{hPa})$ which was not used in GH09. In addition, not only we compute sensitivity fields at $t-48 \mathrm{~h}$ and $t-24 \mathrm{~h}$ but also increase the temporal resolution of the sensitivity fields. Each sensitivity field is computed from 
Table 1. Classification of Mediterranean intense cyclones as derived from the cluster pruning.

\begin{tabular}{|c|c|c|}
\hline Cluster Number & Members & Region \\
\hline 1 & 6 & Gulf of Lions \\
\hline 2 & 28 & Balearic Sea \\
\hline 3 & 43 & Algeria \\
\hline 4 & 27 & \multirow{3}{*}{ South Tyrrhenian Sea } \\
\hline 5 & 12 & \\
\hline 6 & 9 & \\
\hline 7 & 20 & \multirow{2}{*}{ North Tyrrhenian Sea } \\
\hline 8 & 23 & \\
\hline 9 & 20 & \multirow{4}{*}{ Adriatic Sea } \\
\hline 10 & 13 & \\
\hline 11 & 30 & \\
\hline 12 & 24 & \\
\hline 13 & 10 & \multirow{2}{*}{ Sicily } \\
\hline 14 & 8 & \\
\hline 15 & 19 & \multirow{3}{*}{ Ionian Sea } \\
\hline 16 & 13 & \\
\hline 17 & 14 & \\
\hline 18 & 17 & Aegean Sea \\
\hline 19 & 14 & Sea of Crete \\
\hline 20 & 16 & Black Sea \\
\hline 21 & 16 & Turkey \\
\hline 22 & 17 & \multirow{2}{*}{ Cyprus } \\
\hline 23 & 7 & \\
\hline
\end{tabular}

$t-48 \mathrm{~h}$ to $t-6 \mathrm{~h}$ every $6 \mathrm{~h}$. This provides a better monitoring of the evolution of the sensitivity patterns. The precision in the definition of the response function is also improved. GH09 defined the response function for each cluster's member as the average of the MSLP field at time $t$ over an area of $300 \times 300 \mathrm{~km}$ centered over the cyclone center of the cluster's MSLP field. Here, we center the response function over each individual cyclone's center. Therefore, we target individual cyclones and consequently the derived sensitivities as opposed to targeting at a specific geographical location not necessarily linked to the cyclone center position of that member of the cluster.

In order to illustrate the sensitivity information derived in this study and to compare these results with those obtained by GH09, the following subsections describe the results from different points of view: Sect. 3.1 quantifies the homogeneity of the clusters, Sect. 3.2 show the improvement achieved by means of cluster pruning and, finally, some global results of the ensemble sensitivity climatology are presented in Sect. 3.3.

\subsection{Clusters characteristics}

In order to evaluate and compare the homogeneity of the clusters derived in this study to those obtained by GH09, we compute for each cluster the mean euclidian distance between each day and the cluster's centroid in the principal components space of some representative atmospheric fields. The selected fields are: $500 \mathrm{hPa}$ geopotential height at $t-48 \mathrm{~h}$ and $t-24 \mathrm{~h}, 200 \mathrm{hPa}$ geopotential height at $t-48 \mathrm{~h}$, $850 \mathrm{hPa}$ temperature at $t-24 \mathrm{~h}$ and the MSLP at the cyclone's mature stage $t$. The mean euclidian distance computed for all clusters of each classification shows that new clusters are in average 18\% more homogeneous than those produced by GH09. Eventhough linearity can be hampered by other factors, an increment of the significance of the linear trends of the sensitivity computations is expected from this homogeneity improvement. For the new classification, the established threshold for the squared correlation coefficients is 0.16 which leaves unaltered those sensitivity estimates with absolute correlation coefficients higher than 0.4. This lower bound represents the 79th percentile of the distribution of all squared correlation coefficients for all variables and levels considered in this study at $t-48 \mathrm{~h}$ and $t-24 \mathrm{~h}$. However, GH09 established a poorer criteria, that is, 0.1 for the squared correlation coefficients, which represent the 88 th percentile of its equivalent distribution. Thus, even setting a more stringent criterion of significance, this study notably increases the number of unmodified trend values. Regarding the mean significant squared correlation coefficients, that is, those values higher than the linear significance threshold, a notable increase with regard to those obtained by GH09 is derived (Fig. 3). Therefore, in addition to increasing the number of significant sensitivity trends there is also a higher degree of linearity of the unaltered sensitivity estimates. The gradual decline of the linear regime is indicated by weaker linear correlations at earlier lead-times, clearly illustrated now with the increase of the temporal resolution of the sensitivity calculations (Fig. 3).

\subsection{Effects of cluster pruning}

Clusters are pruned for the sake of optimum classes for statistical climatological sensitivity calculation. Here, we illustrate the sensitivity improvement derived from cluster pruning with the Adriatic Sea cyclone class, which is made up of 30 members after pruning. We also test the sensitivity fields corresponding to the original 51-member version (not pruned) of this cluster. The pruned 30-member mean sensitivity field at $t-48 \mathrm{~h}$ shows a main sensitivity structure targeting the strong westerly flow. However, the original 51member mean sensitivity field slightly highlights this region and produces a stronger structure downstream of the westerlies, which unlikely captures the mechanisms involved in the cyclone intensification and are probably produced by spurious distant correlations (Fig. 4). 


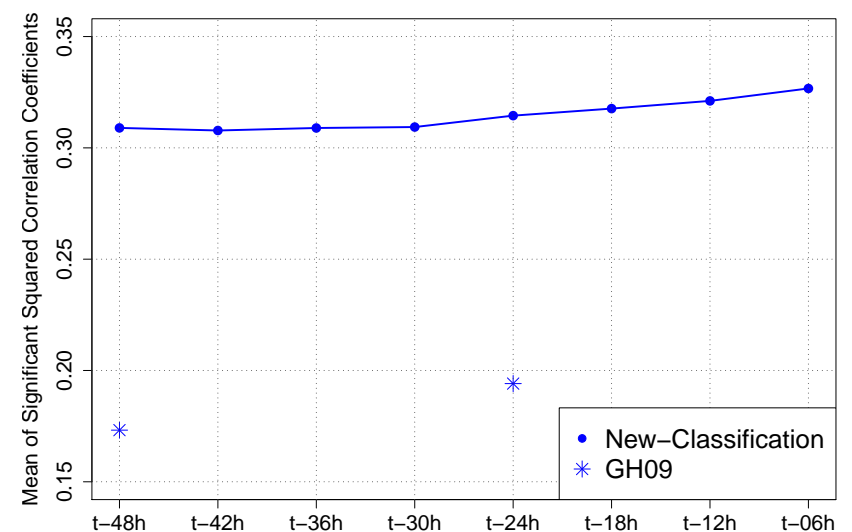

Fig. 3. Time serie of the mean of the significant squared correlation coefficients obtained for all variables and levels considered in this study and its equivalent obtained by GH09.

In order to objectively quantify the accuracy of each sensitivity field, a verification experiment is designed. The verification testbed aims at evaluating the impact on the forecast cyclogenesis of perturbations to the initial conditions over the highlighted sensitivity regions. Errors over these regions are expected to grow the fastest within the linear regime and have a major impact on the mature cyclone prediction. Five days belonging to both clusters are used as common verification sample cases. In addition, we also test the sensitivity fields obtained by GH09 for those clusters to which these 5 episodes belong.

For each case study and sensitivity field to test, a cylindrical gaussian perturbation is applied to the initial conditions $48 \mathrm{~h}$ prior to the maturity of the cyclone. The amplitude of the perturbation is proportional to the mean of the zonal standard deviation of each initial condition field and level. Although the perturbing method does not guarantee the dynamical balance between mass and wind fields, the perturbation amplitudes are small enough to ensure the evolution of the perturbations in the linear regime, minimizing the impact of spurious balancing effects in the verification runs. Regarding the center of the perturbations, it is selected as the grid point with the maximum value of the integral estimate of the mean sensitivity field weighted by a gaussian function (indicated with * in Fig. 4). Thus, in a strict sense, the region where the gaussian perturbation is expected to maximize the impact on the response function is under evaluation. It is worth noting that these test regions are located over sensitivity structures computed from highly significant trend values (above 95\% significance of the trends, indicated with dotted grid points in Fig. 4). Besides the three sensitivity experiments for each case study -based on the pruned and non-pruned mean sensitivity fields and the corresponding mean sensitivity fields obtained by GH09-, a control simulation, without any perturbation, is run. All numerical experiments are performed with the NCAR Advanced Research WRF ARW model (Skamarock et al., 2008) using reanalysis

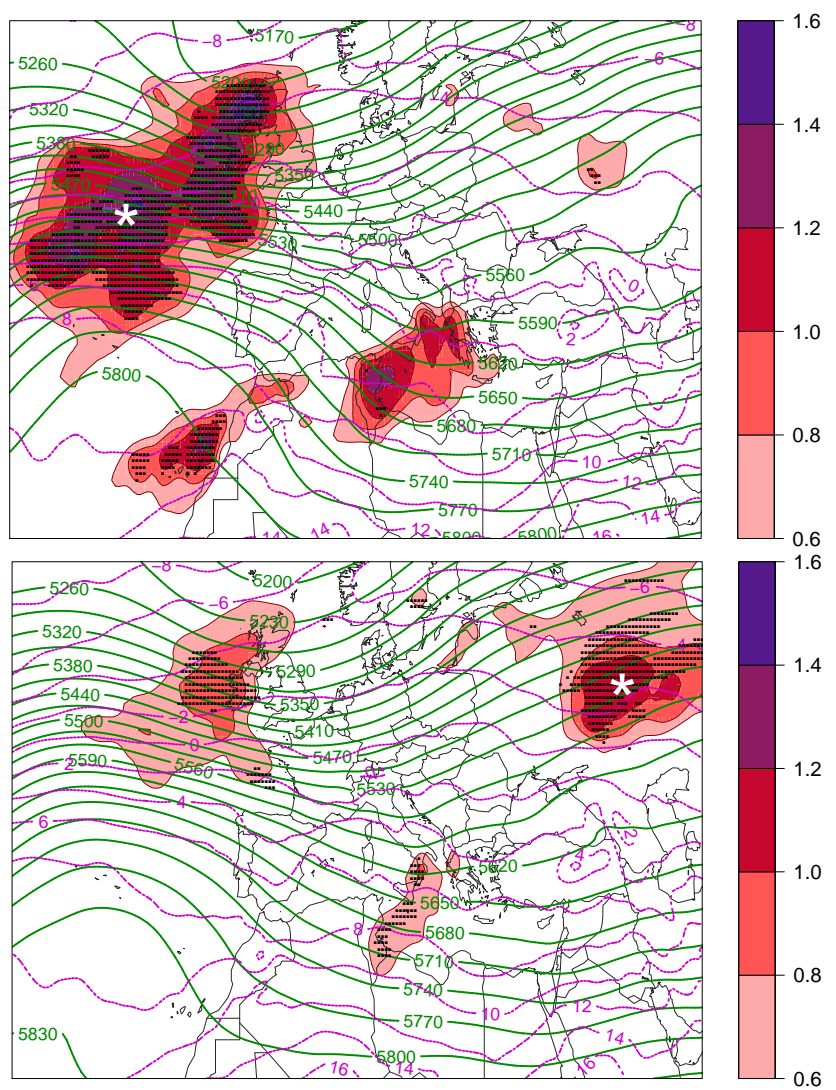

Fig. 4. Mean ensemble sensitivity field (mb,shaded) for an Adriatic Sea cluster at $t-48 \mathrm{~h}$ for the pruned version (30 members, upper panel) and for the non-pruned version (51 members, lower panel). Geopotential height field at $500 \mathrm{hPa}$ (gpm, solid lines) and temperature field at $850 \mathrm{hPa}\left({ }^{\circ} \mathrm{C}\right.$, dashed lines) averaged over all cluster's members. Dots indicate gridpoints where an outstanding percentage of computed trends are above the $95 \%$ significance level. The center of the perturbation of the corresponding numerical experiment is labelled by *.

fields from ERA-40 to initialize the model and to provide the time-dependent boundary conditions. Bearing in mind that all sensitivity fields are derived using a cyclone's depth measure as the response function, the statistical measure used to address the information derived from the simulations should take into account the impact of each perturbation in the cyclone's depth. Therefore, we compute the root mean square of the difference (RMSD) between the MSLP field obtained by a perturbed simulation with respect to the control one. Specifically, this index is computed around the cyclone center. A higher value of RMSD indicates a larger impact of the perturbation over the cyclone's depth. The RMSD is computed every $6 \mathrm{~h}$ during the $48 \mathrm{~h}$ simulation period to provide a more precise monitoring of the impact of each perturbation and therefore to test the time-consistency of the signal. Taking into account that the dynamics involved in each cyclone case are different, a normalization of the RMSD to its maximum value is applied at each time step and case study. 


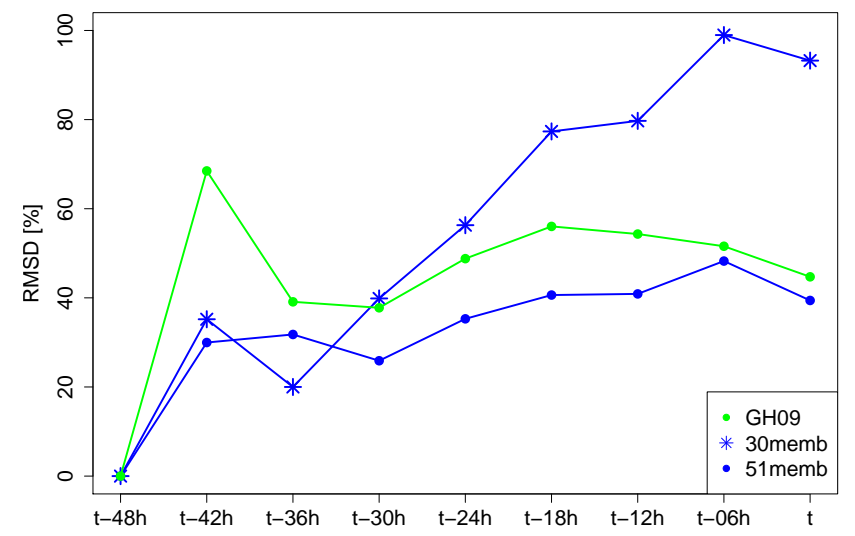

Fig. 5. Time serie of the mean normalized RMSD of the MSLP field between perturbed and control simulations over 5 verification case studies.

Then, the mean of the normalized RMSD index for each method and time step is computed over the 5 events (Fig. 5).

In a strict sense, the derived sensitivity fields are informative about changes in the cyclone depth at its maturity stage produced by a perturbation in the precursor conditions so the RMSD is more representative at the final time steps. Even though the initial evolution of the gaussian perturbation is unstable and undergoes a balancing spin-up period -about $12 \mathrm{~h}$ for all three methods, the balanced part of the perturbations remains and evolves according to the atmospheric dynamics of the day. As shown in Fig. 5, the pruned sensitivity perturbation achieves superior average deepenings for this cluster class than non-pruned and GH09 after the first $24 \mathrm{~h}$ of simulation. Eventually, the best performance in identifying sensitivity areas is obtained with the pruned cluster made up of 30 members with not only a consistent improvement over the more populated cluster of 51 members but also over the sensitivity fields obtained for these case studies by GH09. This is arguably attributed to the better cluster homogeneity achieved with the pruning process which is essential to approach the statistical sensitivity method hypothesis and thus to improve the reliability of the resulting sensitivities.

\subsection{Global Results}

For each of the classes listed in Table 1, sensitivity fields are computed for all considered precursor conditions and levels and the complete detailed sensitivity climatology is derived. At this point, the effects of reduced sample size becomes evident for clusters such as \#1 Gulf of Lions (6 members), \#5 and \#6 South Tyrrhenian (12 and 9 members) or \#13 Sicily (10 members). Average sensitivity maps for these clusters show widespread sensitivity structures most likely originated from spurious correlations which progressively fade away as the cluster size increases. Note that the minimum number of members in a cluster to obtain an acceptable signal to spurious noise ratio is highly dependent on the cluster topology and the dynamical evolution of the cyclogenesis type.
Here, a pragmatic approximation is used and this lower limit is chosen subjectively by individually analyzing each of the 23 summary cluster-averaged sensitivity fields. All in all, clusters larger than 13 members show reasonable sensitivity structures and so will be accounted for in further analysis. Sensitivity results for cyclone classes with less than 14 members are discarded. These cyclone types are too rare for current available catalogs (based on ERA-40) to properly isolate significant sensitivity structures using the statistical sensitivity method.

For the sake of brevity, only summary sensitivity maps over all cyclone types are shown for each time step (Fig. 6). These fields provide a general outline of the regions which are persistently visited by atmospheric features that influence the genesis and deepening of Mediterranean intense cyclones. In order to account for the different frequency of each cluster and the significance of each cyclone sensitivity field, these mean fields are calculated as the average of the linear trends times the standard deviation, and are weighted by the population of each cluster and the local squared correlation coefficient in the database. As a consequence of the gradual decrease in the correlation coefficients (Fig. 3), there is a fading-out of the resulting sensitivity fields at earlier time steps due to the progressive degradation of the linear assumption. The further back we compute sensitivities, the weaker the significant linear sensitivity results.

For longer ranges, the climatology shows sensitivity areas over eastern North African lands, parts of central Mediterranean, central Europe and the European Atlantic coasts (Fig. 6). As we get closer to the hazardous cyclone maturity, the Atlantic, central Europe and central Mediterranean become increasingly relevant, with a notable contraction of the sensitive structures towards the central Mediterranean. These results are remarkably similar to the GH09 fields (Fig. 7), specially for $t-24 \mathrm{~h}$, when sensitivities highlight the continental Europe and the western and central Mediterranean basins. This particular division is consistent with previous results that highlight the western north Africa and the central Mediterranean as areas with high density of Mediterranean cyclone trajectories (Lionello et al., 2002) and also reveals the crucial role of mid-level troughs across Europe and arriving from the Atlantic storm track on the last $24 \mathrm{~h}$ before cyclone maturity. For the last stages before maturity, the sensitivity structure is compacted around the Mediterranean basin and central Europe as an obvious consequence of the effect of surrounding environments have at the last cyclogenetic stages. It is worth mentioning that the increased temporal resolution of these results compared to those in GH09 allows for a better monitoring of the evolution of the sensitivity structures. The temporal tracking of the signal is also carried out by other ensemble-based techniques such as Ensemble Transform Kalman Filter (Bishop et al., 2001; Majumdar et al., 2010) as it is a valuable information which allows to discriminate between targets that are genuine and those that are spurious. 

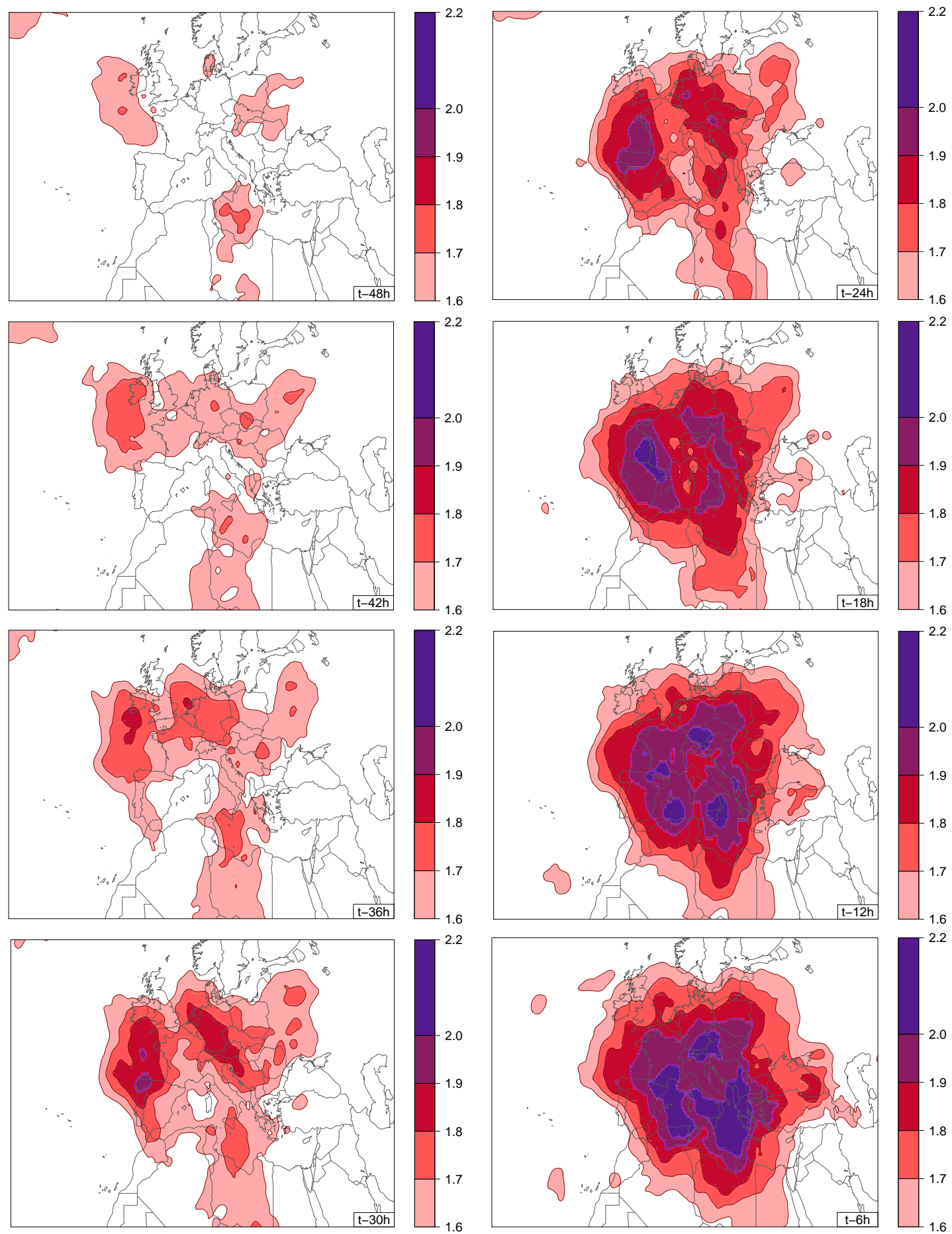

Fig. 6. Mean sensitivity fields for sufficiently populated clusters computed over all considered precursor conditions and levels (mb, shaded) from $t-48 \mathrm{~h}$ to $t-6 \mathrm{~h}$ every $6 \mathrm{~h}$ (in order from left to right and top to bottom).

\section{Conclusions}

After the astute sensitivity calculation method proposed by Hakim and Torn (2008), which allows to easily derive sensitivity fields from collections of slightly differing atmospheric evolutions, a door was opened in the field of sensitivity cli- matologies. Information derived from such sensitivity analyses provide guidance for decision makers regarding the future design of systematic and targeted observational platforms. Garcies and Homar (2009) proved the applicability of the method to produce a climatology of sensitivities 


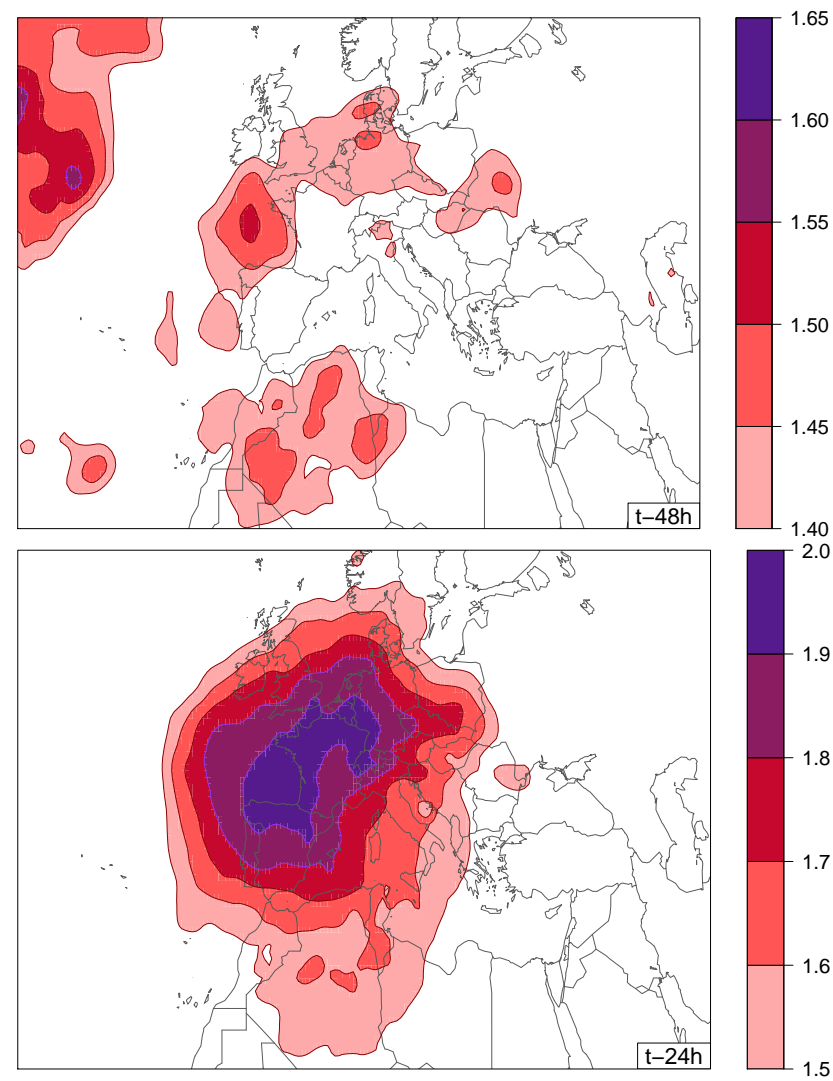

Fig. 7. Mean sensitivity fields obtained by GH09 for 25 cyclones types computed over all considered precursor conditions and levels (mb, shaded) for $t-48 \mathrm{~h}$ (upper panel) and $t-24 \mathrm{~h}$ (lower panel). Note the scale change between figures. (Updapted from GH09).

for Mediterranean intense cyclones using the catalog of intense cyclones derived by Jansà and Homar (2006). The ultimate goal of these researchs is to improve upon current forecasts of extremely hazardous weather episodes. Admittedly, the climatological analyses used for these studies do not resolve the mesoscale and sub-mesoscale phenomena directly responsible for the severe impacts; however, it is an underlying hypothesis of our investigations that getting better forecasts of the parent system characteristics will necessarily also improve the forecasts of smaller scale potentially damaging features within the synoptic cyclone.

This paper reports on the results of the natural extension of previous findings. Once the statistical sensitivity calculation method is proven to produce sensible results when used on already available clusters of Mediterranean cyclones, the quality of the final products is expected to improve when an ad-hoc catalog of Mediterranean intense cyclones is built. The new catalog contains 406 episodes classified into $23 \mathrm{cy}-$ clone types covering 13 regions across the Mediterranean basin. Besides the new classification process, the homogeneity of the classes is maximized using a pruning strategy that significantly increases the accuracy of the result- ing sensitivity fields. This outstanding improvement is confirmed by numerical experiments which show significantly higher responses on the forecasted cyclone for a pruned cluster than for both the non-pruned version or the previous results (GH09). In addition to that, various typological indicators reveal the superior quality of the catalog of cyclones used to derive the new climatology of sensitivities. For instance, the correlation between precursing conditions and the response function is higher than in the previous climatology. Taking advantage of that, the criteria to smooth-out high sensitivity signals in low correlated areas is now more restrictive (threshold of 0.4 correlations as opposed to 0.32 in GH09) and even then, the number of significant trends is notably increased (60\% above) in this new catalog.

The optimized catalog of intense cyclones for statistical sensitivity calculation is obtained at the expense of sample size. Some cyclone classes in the catalog contain too few members for the statistical results to be fully reliable and allow for a clear signal/noise detection of the sensitivity. This drawback is an explicit limitation of the method when applied on unfrequent events (intense cyclones) within a finite size timespan (ERA-40 period: from 1957 to 2002). Future databases that cover longer periods will undoubtedly overcome this handicap. In face of minimizing the impact of such potentially inaccurate partial results, clusters with less than 14 members are not considered when computing general synthetic sensitivity fields (i.e., 328 cyclones belonging to 15 different cyclone types are considered for summary sensitivity maps). Also, these primary mean sensitivity results are weighted by cluster size and average correlation coefficient so that larger (more frequent) clusters and more linear cyclones produce higher impact onto the mean fields.

The general results hold two interesting characteristics. On the one hand, although final products may admittedly miss relevant undersampled sensitivity patterns due to lowcorrelation areas being smoothed-out from the results, structures not filtered out by the method show robust sensitivity information. This important property grants reliability to a product ultimately oriented to provide guidance on actual decisions regarding deployment and relocation of observational means. On the other hand, together with the fading effect, sensitivity patterns tend to move away from the Mediterranean as they are computed further back in time. This agrees with the intuitive idea of a spatiotemporal cone of events that can influence the mature cyclone formation.

Regarding the location of the main sensitivity areas, the averaged results highlight the European Atlantic coasts, central and western Europe, the central and western Mediterranean basin and north African lands as regions where important atmospheric structures for the short-range forecast of Mediterranean intense cyclones are persistently located. Reinforcing the quality and capacity of observation means over these areas would benefit the numerical weather prediction of these hazardous systems in the sense that the sensitivity fields indicate the regions where errors in the initial 
conditions grow the fastest, causing large forecast failures when not observed.

Finally, the reliability of these climatological sensitivity results can be further quantified by means of further verification experiments using numerical weather prediction systems. Such verification testbed would confirm not just the quality of the linear sensitivity results but their applicability for the short-range prediction of relevant aspects of Mediterranean high-impact weather. This remains for future research.

Acknowledgements. The ECMWF is acknowledged for providing the ERA-40 reanalysis. The authors also thank the Mediterranean Studies Section of the Centro Meteorológico Territorial en Illes Balears of the Spanish Weather Service (AEMET) for building the cyclone databe. This research has been supported by MEDICANES project (CGL2008-01271/CLI). L. Garcies also acknowledges support from Spanish MEC through FPU grant (AP2007-01367).

Edited by: M.-C. Llasat

Reviewed by: two anonymous referees

\section{References}

Ancell, B. and Hakim, G. J.: Comparing Adjoint- and EnsembleSensitivity Analysis with Applications to Observation Targeting, Mon. Wea. Rev., 135, 4117-4134, 2007.

Anderberg, M. R.: Cluster Analysis for Applications, Academic Press, New York, 1973.

Bishop, C. H., Etherton, B. J., and Majumdar, S. J.: Adaptative sampling with the Ensemble Transform Kalman Filter. Part I: Theoretical Aspects, Mon. Wea. Rev., 129, 420-436, 2001.

Campins, J., Jansà, A., and Genovés, A.: Three-dimensional structure of Western Mediterranean cyclones, Int. J. Clim., 26, 323343, 2006.

Campins, J., Genovés, A., Picornell, M., and Jansà, A.: Climatology of Mediterranean cyclones using the ERA-40 dataset, Int. J. Climatol, p. Published online in Wiley InterScience (www. interscience.wiley.com), 2010.

Dunn, J. C.: Well separated clusters and optimal fuzzy partitions, J. Cybern., 4, 95-104, 1974.

Garcies, L. and Homar, V.: Ensemble sensitivities of the real atmosphere: application to Mediterranean intense cyclones, Tellus, 61A, 394-406, 2009.

Hakim, G. J. and Torn, R. D.: Ensemble Synoptic Analysis, Sanders Symposium Monograph, Meteor. Monogr., 55, Amer. Meteor. Soc., in press., 2008.
Halkidi, M., Batistakis, Y., and Vazirgiannis, M.: On Clustering Validation Techniques, Journal of Intelligent Information Systems, 17, 107-145, 2001.

Homar, V., Jansà, A., Campins, J., and Ramis, C.: Towards a climatology of sensitivities of Mediterranean high impact weather - first approach., Advances in Geosciences, 7, 259-267, 2006.

Homar, V., Jansà, A., Campins, J., Genovés, A., and Ramis, C.: Towards a systematic climatology of sensitivities of Mediterranean high impact weather: a contribution based on intense cyclones, Natural Hazards and Earth System Sciences, 7, 445-454, 2007.

Jansà, A. and Homar, V.: Climatology of sensitivities of high impact weather in the Mediterranean, EUCOS REPORT, EUCOS Studies Programme. Reading, UK, 2006.

Jansà, A., Genovés, A., Picornell, M. A., Campins, J., Riosalido, R., and Carretero, O.: Western Mediterranean cyclones and heavy rain, Meteorol. Appl., 8, 43-56, 2001.

Jollife, I. T.: Principal Component Analysis, Springer Series in Statistics, Springer, New York, 2nd edn., 2002.

Lionello, P., Dalan, F., and Elvini, E.: Cyclones in the Mediterranean Region: the present and the doubled $\mathrm{CO}_{2}$ climate scenarios, Clim. Res., 22, 147-159, 2002.

Majumdar, S. J., Sellwood, K. J., Hodyss, D., Toth, Z., and Song, Y.: Characteristics of Target Areas Selected by the Ensemble Transform Kalman Filter for Medium-Range Forecasts of High-Impact Winter Weather, Mon. Wea. Rev., 138, 2803-2824, 2010.

Marseille, G. J. and Bouttier, F.: From sensitivity studies to observation planning, EUCOS REPORT, EUCOS-REP-030. Reading, UK, 2000.

Milligan, G. and Cooper, M.: An examination of procedures for determining the number of clusters in a data set, Psychometrika, 50, 159-179, 1985.

Richman, M. B.: Rotation of principal components, J. Climatol., 6, 293-335, 1986.

Skamarock, W. C., Klemp, J. B., Dudhia, J., Gill, D. O., Barker, D. M., Duda, M., Huang, X.-Y., Wang, W., and Powers, J. G.: A Description of the Advanced Research WRF Version 3, Tech. rep., NCAR Tech. Note NCAR/TN-475+STR, Boulder, USA, 2008.

Uppala, S. M., Kållberg, P. W., Simmons, A. J., Andrae, U., da Costa Bechtold, V., Fiorino, M., Gibson, J. K., Haseler, J., Hernandez, A., Kelly, G. A., Li, X., Onogi, K., Saarinen, S., Sokka, N., Allan, R. P., Andersson, E., Arpe, K., Balmaseda, M. A., Beljaars, A. C. M., van de Berg, L., Bidlot, J., Bormann, N., Caires, S., Chevallier, F., Dethof, A., Dragosavac, M., Fisher, M., Fuentes, M., Hagemann, S., Holm, E., Hoskins, B. J., Isaksen, L., Janssen, P. A. E. M., Jenne, R., McNally, A. P., Mahfouf, J. F., Morcrette, J. J., Rayner, N. A., Saunders, R. W., Simon, P., Sterl, A., Trenberth, K. E., Untch, A., Vasiljevic, D., Viterbo, P., and Woollen, J.: The ERA-40 re-analysis, Q. J. R. Meteorol. Soc., 131, 2961-3012, 2005. 